\title{
Diagnóstico da inovação em empresas de tecnologia da informação de um município do sudoeste do Paraná
}

\author{
Rosaine Fiorio Semler Acadêmica do Programa de Pós-Graduação em Engenharia de Produção e Sistemas - Universidade \\ Tecnológica Federal do Paraná (UTFPR) - Brasil, rosainefiorio@gmail.com \\ Fernando José Avancini Schenatto Doutor em Engenharia de Produção, Universidade Tecnológica Federal do Paraná (UTFPR) - Brasil, \\ schenatto@utfpr.edu.br
}

\section{RESUMO}

O ciclo de vida de produtos e tecnologia tem-se encurtado principalmente devido à alta competitividade do ambiente de negócios e, nesse sentido, a capacidade de lidar com essas dimensões da gestão empresarial é crucial para a competitividade das organizações, principalmente as empresas intensivas em tecnologia, como é o caso da indústria de software. Dessa forma, o objetivo desse estudo é mensurar as dimensões da inovação e estagiar a inovação nas empresas que compõem um núcleo de tecnologia. Sendo assim, tem-se um estudo exploratório, multicascos, com a aplicação de dois questionários sendo o primeiro proposto por Carvalho, Reis e Cavalcante (2011) que visa avaliar maturidade das empresas frente à inovação e o segundo de Scherer e Carlomagno (2009) que analisa as dimensões da inovação, sendo ambos complementares. Como resultados obteve-se quanto aos estágios de inovação que três empresas estão no estágio Disseminação, uma em Inovação como Estratégia; três como Estruturação e três como Parcerias e quanto às dimensões destaca-se funding e processo como deficitárias e como mais desenvolvida a dimensão Estrutura. Para tanto, na intersecção entre os resultados dos dois métodos pode-se estabelecer ações para melhoria da inovação nessas empresas.

Palavras-chave: Inovação. Maturidade da Inovação. Dimensões da Inovação. Empresas de TI.

\section{Diagnosis of innovation in information technology companies of a municipality in the southwest of Paraná}

\begin{abstract}
The life cycle of products and technology has shortened mainly due to highly competitive business environment and in this sense, the ability to deal with these dimensions of business management is crucial to the competitiveness of organizations mainly intensive companies in technology, such as in the case of software industry. Thus, the aim of this study is to measure the dimensions of innovation and the stage of innovation in enterprises that form a technological center. Thus, this is an exploratory study of multicases with the application of two questionnaires: the first proposed by Carvalho, Reis and Cavalcante (2011) that aims to evaluate the maturity of companies facing innovation and the second by Scherer and Carlomagno (2009) that analyzes the dimensions of innovation, both being complementary. The result obtained showed that regarding innovation, three companies are in the Dissemination stage, one in Innovation as Strategy; three in Structuring and another three in Partnerships. Concerning the dimensions, funding and processes stand out as in deficit. As more developed dimension, it is the structure dimension that stands out. Therefore, at the intersection of the results of the two methods, actions to improve innovation in these companies can be established.
\end{abstract}

Keywords: Innovation. Maturity of Innovation. Dimensions of Innovation. IT companies. 


\section{INTRODUÇÃO}

O mercado de tecnologia da informação tem características singulares: baixo custo de entrada de novos players no mercado; produtos em constante evolução; e baixa apropriabilidade, pois, durante o desenvolvimento, a etapa de análise consome recursos e tem grande importância, assim, o software é subproduto das empresas que, copiado isoladamente, não trará vantagem competitiva a quem o copiou (BRESCHI; MALERBA, 1997). Isto em um setor de alta competitividade fazem da inovação fator essencial de sobrevivência.

Para se manter em um mercado competitivo, acirrado e altamente tecnológico, são necessárias estratégias de inovação (MIOZZO; SOETTE, 2001). Contudo, uma organização inovadora possui habilidade de criar valor superior ao cliente e, como consequência, estas habilidades são comparadas aos competidores posicionando-a no segmento de mercado e incentivando a busca obsessiva no aumento do valor aos clientes (KNOX, 2002). Assim, o processo de inovação e gestão da inovação ultrapassa o investimento em tecnologia, estando este ligado ao desenvolvimento e implantação de novidades, incluindo processos, ideias, tecnologias, produto ou arranjos (VAN DE VEN et al, 1999; BARBIERI; ÁLVARES, 2004).

Apesar disso, poucos estudos têm foco no segmento de mercado de Tecnologia da Informação, principalmente com vista à inovação e, segundo Marques, Suzuki e Faria (2010), é imprescindível a realização de estudos do processo de inovação, dispondo dados de forma a auxiliar empreendedores na gestão das empresas de tecnologia da informação.

Com isso, até o momento, não há estudo formal publicado com diagnóstico da inovação em empresas de TI do Sudoeste do Paraná, especificamente as empresas participantes do núcleo de empresas do setor de TI situado em Francisco Beltrão - PR. Assim, este estudo busca expor dados do nível de maturidade em gestão da inovação de empresas desse núcleo, resultando uma ferramenta de construção de conhecimento, visto que são poucos os estudos voltados para a inovação em empresas de $\mathrm{Tl}$, em especial as microempresas, abordando temas de estágio e dimensionamento da inovação. São também apresentados dados relevantes, de identificação da evolução e pontos fortes e fracos relacionados à inovação dessas empresas.

\section{FUNDAMENTAÇÃO TEÓRICA}

Nessa seção serão apresentados os conceitos que permearam a pesquisa a saber: inovação, a avaliação e o dimensionamento da inovação e uma caracterização da indústria de software como componente do setor de TI.

\section{1 Inovação}

O termo "inovação" introduzido por Joseph Alois Schumpeter, na obra intitulada "The Theory of Economic Development", em 1934, na análise econômica, sendo definida como algo novo sendo aplicado comercial ou industrialmente, seja sob a forma de produtos, processos, meios de produção, suprimentos ou demais insumos; e trazia em seu âmago uma nova forma de organização de negócios e mesmo de relações de trabalho (SCHUMPETER, 1934).

Uma importante referência para a definição de inovação encontra-se no Manual de Oslo, publicado pela OCDE (Organização para a Cooperação e Desenvolvimento econômico), de forma a orientar e padronizar conceitos, metodologias e construção de estatísticas e indicadores de pesquisa de inovação na indústria, define inovação como: "a implementação de um produto, bem ou serviço novo ou significativamente melhorado ou um processo ou um novo método de marketing ou um novo método organizacional nas práticas de negócios na organização do local de trabalho ou nas relações externas" (OCDE, 2006, p. 55).

Segundo PINTEC (2011), empresas de pequeno porte mantêm índices de inovação inferiores aos das empresas de grande porte: entre 2006 e 2008 somente 38\% de micro e pequenas empresas geraram alguma inovação, já $72 \%$ das empresas de grande porte criaram algum produto ou processo. No entanto, a maioria das MPEs não tem conhecimento da importância da inovação para garantia de funcionamento e permanência no mercado.

O financiamento pode ser fator determinante para inovação, de acordo com o Manual de Oslo, entretanto MPEs enfrentam dificuldades ao obter financiamento externo, sendo este um ponto de obstrução para atividades inovativas, juntamente com insuficiência de pessoal qualificado para empreender inovação; dificuldades para encontrar este pessoal no mercado; ausência de infraestrutura; falta de conhecimento sobre tecnologia ou mercado; e incapacidade de encontrar parceiros apropriados para projetos conjuntos de inovação (OCDE, 2006; SUH; WANG, 2010; VEE, 2010; SOUZA BERMEJO, 2015). De encontro a essas afirmações, Caron (2004) elenca, em ordem de 
importância, como sendo dificuldade enfrentadas pelas MPEs para inovar: falta de recursos para investimentos; acesso a financiamentos; informações sobre entidades de apoio à inovação tecnológica; recursos humanos capacitados; máquinas e equipamentos; informações sobre mudanças tecnológicas; confiança em parcerias e alianças para inovação tecnológica; e informações sobre mercados.

Kleinknecht (1987), Kleinknecht e Reijenen (1991) e Brouwer e Kleinknecht (1997), constatam que MPEs têm atividades inovativas não formais ou não explícitas, criando um viés para baixo nas medidas de propensão a inovação. Vaona e Pianta (2008) relatam que muitas das inovações em processos de MPEs estão relacionadas à incorporação de capital físico ao invés de investimentos tangíveis em pesquisa e desenvolvimento

Com isso, faz-se necessário que as empresas, especificamente para esse estudo do setor de TI, tenham uma avaliação da sua capacidade inovativa baseando-se em diferentes indicadores que possam expressar o seu grau de inovação, como o estagiamento e o conhecimento de suas fortalezas e fraquezas em relação as dimensões que compõem a inovação.

\section{2 Avaliação e dimensionamento da inovação}

O Quadro 1 lista metodologias com fim de avaliar o grau de inovação das organizações, cada uma com foco em um aspecto ou um conjunto de aspectos da gestão da inovação.

\begin{tabular}{|c|c|c|c|}
\hline \multicolumn{4}{|c|}{ Quadro 1- Metodologias de Avaliação da Inovação } \\
\hline Ano & Autores & Dimensões & Características \\
\hline 1990 & Griliches & Não há. & $\begin{array}{c}\text { Defende a tese de que o grau de inovação de uma empresa } \\
\text { está diretamente ligado ao número de patentes que } \\
\text { publicou. }\end{array}$ \\
\hline 2001 & Shapiro & & $\begin{array}{c}\text { Traz seis estágios de inovação: - funcionalmente vinculada, } \\
\text { - sensível a processos, - orientada a processos, - dominada } \\
\text { por processos, - baseada em capacidade e baseada em } \\
\text { aliança. }\end{array}$ \\
\hline 2006 & Sawhney & $\begin{array}{c}12 \text { Dimensões: oferta, } \\
\text { plataforma, marca, clientes, } \\
\text { soluções, relacionamento, } \\
\text { agregação de valor, processos, } \\
\text { organização, cadeia de } \\
\text { fornecimento, presença e rede. }\end{array}$ & $\begin{array}{l}\text { Classifica a empresa como pouco ou nada inovadora, } \\
\text { inovadoras ocasionais ou inovadoras sistêmicas. }\end{array}$ \\
\hline 2007 & Mackinnons & $\begin{array}{l}\text { Modelo das } 7 \text { dimensões da } \\
\text { inovação. }\end{array}$ & $\begin{array}{l}\text { Os estágios de maturidade em gestão da inovação são } \\
\text { divididos em quatro estágios: ad hoc, onde a inovação não } \\
\text { é gerenciada sistematicamente; inovação localizada, onde } \\
\text { a inovação tem o gerenciamento eficiente em algumas } \\
\text { áreas; inovação generalizada, em que há introdução de } \\
\text { processos e estruturas para gerenciamento da inovação; e a } \\
\text { inovação contínua, estágio em que as organizações } \\
\text { realizam avaliações contínuas e aprimoram sua } \\
\text { performance. }\end{array}$ \\
\hline 2008 & $\begin{array}{c}\text { Bachmann e } \\
\text { Destafani }\end{array}$ & $\begin{array}{l}12 \text { dimensões de Sawhey } \\
\text { (2006) com inclusão de } \\
\text { ambiência inovadora }\end{array}$ & $\begin{array}{c}\text { Metodologia baseada nas doze dimensões da inovação } \\
\text { propostas por Sawhney (2006). }\end{array}$ \\
\hline 2009 & INEI & & $\begin{array}{l}\text { Modelo de avaliação da inovação baseado na aplicação do } \\
\text { software QDI (Questionário Diagnóstico de Inovação) que } \\
\text { analisa os fatores críticos do processo de inovação. }\end{array}$ \\
\hline 2009 & $\begin{array}{c}\text { Scherer e } \\
\text { Carlomagno }\end{array}$ & $\begin{array}{l}\text { Oito dimensões avaliadas: } \\
\text { liderança, estratégia, } \\
\text { relacionamento, cultura, } \\
\text { pessoas, estrutura, processo e } \\
\text { funding }\end{array}$ & $\begin{array}{l}\text { Propõem a utilização do octógono da inovação como } \\
\text { ferramenta de diagnóstico do potencial inovador, assim } \\
\text { como para a gestão de empresas inovadoras. }\end{array}$ \\
\hline 2011 & $\begin{array}{l}\text { Cavalcante, } \\
\text { Carvalho e } \\
\text { Reis }\end{array}$ & & $\begin{array}{l}\text { São utilizados quatro conjuntos de indicadores: esforços da } \\
\text { empresa voltados à inovação, processos de gestão da } \\
\text { inovação, estímulo à inovação e resultados advindos da } \\
\text { inovação, a partir dos quais classifica a inovação na } \\
\text { organização em um dentre oito estágios: conscientização, } \\
\text { disseminação, inovação como estratégia, parcerias, } \\
\text { estruturação, processo de gestão da inovação, capacidade } \\
\text { de inovar e acompanhamento. }\end{array}$ \\
\hline
\end{tabular}

Fonte. Elaborado pelos autores (2016) 
Enquanto o modelo de Griliches (1990) foca o quantitativo de patentes e o proposto por INEI (2009) analisam fatores ligados à inovação, os demais consideram fatores externos e internos à organização, propondo a análise de componentes organizacionais tratados como dimensões, importantes componentes da inovação (SAWHNEY et al, 2006; MACKINNON, 2007; BACHMANN; DESTAFANI, 2008; SCHERER; CARLOMAGNO, 2009), outros classificam a inovação dentro de uma escala de estágios (SHAPIRO, 2001).

Os modelos de Scherer e Carlomagno (2009) e Carvalho, Reis e Cavalcante (2011) se complementam. No primeiro, o resultado expressa dimensões da empresa em seu esforço inovativo; e o segundo classifica a empresa em seu estágio de inovação. Ambas as metodologias permitem a obtenção dos objetivos deste trabalho.

\section{3 O segmento de desenvolvimento de software na indústria de tecnologia da informação}

A "indústria de tecnologia da informação e comunicação" surgiu dos computadores e telecomunicações, sendo que a de computadores desmembrou-se em dois componentes: software e hardware, sendo sua linha histórica permeada pelos avanços da microeletrônica e telecomunicações (SCHENATTO, 2012).

No Brasil, o fomento às tecnologias da informação por meio de políticas governamentais é recente. Entre 1992 e 1995, a política de reserva e proteção do mercado interno foi substituída por outra visando fomentar a inserção brasileira no mercado mundial (DUARTE; BRANCO, 2001).

Ao mesmo tempo foi estabelecido, sob direção do Conselho Nacional de Desenvolvimento Científico e Tecnológico (CNPq/MCT), o Programa Nacional de Software para a Exportação (SOFTEX), (GRIZENDI, 2012) objetivando executar, promover, fomentar e apoiar ações de inovação, desenvolvimento e exportação do software brasileiro. O setor de software é intensivo em tecnologia e conhecimento, tem características de grande necessidade de inovações técnicas, alta competitividade, baixo investimento de capital fixo e dependência da capacidade criativa e intelectual de colaboradores (MILESI; PETELSKI; VERRE, 2013; FRANK et al, 2016; MIOZZO, et al, 2016).

As diferentes e novas tecnologias disponibilizadas através do setor de TI alteram o contexto em que se inserem ao mesmo tempo que propiciam conhecer esse novo ambiente por elas mesmas. Além disso, nesse setor, para Malerba e Orsenigo (1993, p. 60 apud SCHENATTO, 2012, p. 60), "dada à complexidade dos conhecimentos necessários à atividade inovadora e a heterogeneidade de competências, a cooperação entre as firmas e as estratégias de rede são muito amplas".

\section{METODOLOGIA}

A investigação do tema de pesquisa "Diagnóstico da inovação em empresas de tecnologia da informação em Francisco Beltrão - PR" foi organizada a partir de sua finalidade descritiva, onde, segundo Vergara (2000), o objetivo desse tipo de pesquisa é descobrir as principais características relativas à população pesquisada. A estratégia ou procedimento da pesquisa pode ser definida como levantamento ou survey (BABBIE, 1999), vista a realização de estudo aos indicadores relativos à inovação em empresas de TI, onde o instrumento utilizado foi um questionário estruturado.

O objetivo deste trabalho é identificar o nível de maturidade da gestão da inovação em empresas de TI da cidade de Francisco Beltrão - PR. O universo pesquisado constitui-se das 16 empresas ligadas ao Núcleo Beltronense de Empresas de Tecnologia da Informação. Os sujeitos da pesquisa são os gestores das empresas (Gerentes, Diretores de TI ou CEOs), sendo esses atores detentores das informações necessárias à definição do nível de maturidade da inovação.

Os instrumentos de pesquisa utilizados foram dois questionários, o primeiro proposto por Carvalho, Cavalcante e Reis (2011) - Instrumento 1 - avalia quatro frentes: esforços da empresa voltados à inovação; processo de gestão da inovação; estímulos à inovação e resultados advindos da inovação. Sendo esse composto por questões fechadas com 4 alternativas: Inexiste, Não formalizado, Pouco formalizado e Formalizado. O segundo questionário foi proposto por Scherer e Carlomagno (2009) - Instrumento 2 - composto por 24 frases afirmativas que devem ser pontuadas pelo respondente entre 1 (menor nota) e 9 (maior nota).

Os referidos instrumentos foram aplicados entre os meses de novembro e dezembro de 2015, presencialmente, com os diretores e/ou gestores das empresas, em forma de entrevistas, no ambiente das empresas, resultando em uma amostra de 11 empresas, sendo estas documentadas manualmente. As demais empresas, totalizando cinco, optaram por não participar da pesquisa.

Os dados resultantes receberam os seguintes tratamentos: 
- Instrumento 1: realizado somatório do resultado da avaliação dos aspectos inerentes a esforços, processo, estímulos; e

- Instrumento 2: como as afirmativas que compõem o questionário são classificadas dentro de cada uma das oito dimensões propostas, sendo o resultado obtido através da média das notas atribuídas às questões inerentes à dimensão avaliada.

Então, conforme os modelos, se realizou a comparação quanto à escala de estagiamento e foram elaborados diagramas na forma de octógono com as dimensões consideradas. Para preservação da identidade, as empresas participantes foram representadas por codinomes.

\section{ANÁLISE E DISCUSSÃO DE RESULTADOS}

Essa seção exibe e discute - à luz da literatura - os resultados da aplicação dos métodos em 11 microempresas do setor de TI. A seção é dividida em subseções demonstrando-se a caracterização das empresas, análise de dados frente ao estágio da inovação, representações gráficas com dimensões da inovação e análise confrontando resultados dos dois métodos.

\subsection{Caracterização da empresas}

As empresas participantes atuam na área de $\mathrm{Tl}$, apresentam porte de microempresa, de acordo com classificação proposta pelo BNDES (2011) baseada no faturamento anual. Destas, três têm mais de 15 colaboradores e faturamento entre $\mathrm{R} \$ 500.001$ e $\mathrm{R} \$ 1.200 .000$.

\subsection{Estagiamento da inovação}

O questionário desenvolvido por Carvalho, Cavalcante e Reis, (2009) permite situar o estágio da inovação da organização. As classificações das empresas participantes desse estudo são demonstradas no Quadro 2.

Quadro 2 - Estágio da inovação das empresas em estudo
\begin{tabular}{|cc|}
\hline Codinome & Classificação \\
\hline Superman & Estruturação \\
Lanterna Verde & Estruturação \\
Ciborgue & Estruturação \\
Aquaman & Estruturação \\
Homem de Ferro & Parcerias \\
Gavião Negro & Parcerias \\
Arqueiro Verde & Parcerias \\
Batman & Inovação como estratégia \\
Mulher-Maravilha & Disseminação \\
Capitão América & Disseminação \\
Demolidor & Disseminação \\
\hline
\end{tabular}

Fonte: Elaborado pelos autores (2016).

É possível identificar que somente uma empresa se enquadrou no estágio de Inovação como estratégia, estando as demais em Estruturação, Disseminação e Parcerias.

\section{3 Octógono da inovação}

Os dados da pesquisa foram dispostos em dois gráficos com objetivo exclusivo de facilitar a visualização e análise das informações. Na Figura 1, apresenta-se resultados das empresas com codinome Lanterna Verde, Homem de Ferro, Ciborgue e Superman. 


\section{Figura 1 - Octógono da Inovação Agrupamento 1}

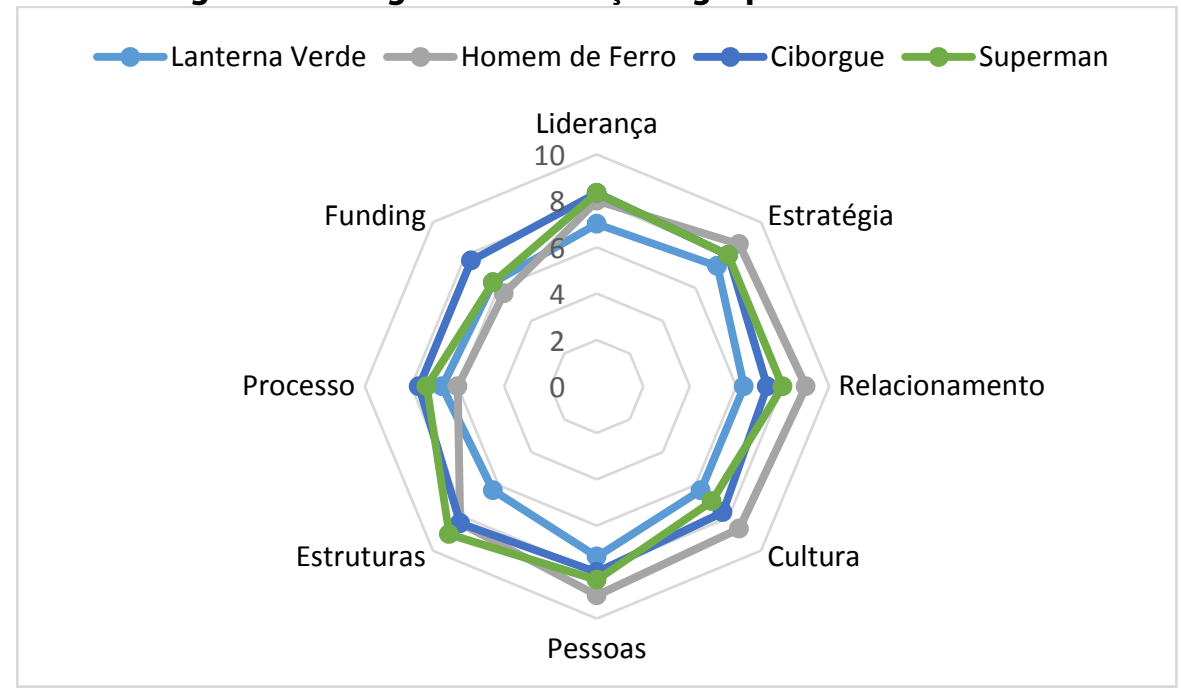

Fonte: Elaborado pelos autores (2016).

Verifica-se que essas empresas apresentam o traçado mais coerente com o octógono e que as dimensões que se mostram fracas são funding, processo e estruturas.

Das empresas, Lanterna Verde e Superman, ambas em estágio de Estruturação da inovação, possuem respectivamente, 83 e 18 colaboradores, e faturamento de $\mathrm{R} \$ 500.001$ a $\mathrm{R} \$$ 1.200.000. Já Homem de Ferro e Ciborgue, em estágio de inovação de Parcerias e Estruturação, têm 2 colaboradores, e faturamento menor (até R\$ 240.000). Os dados corroboram com Kleinknecht (1987); Kleinknecht e Reijenen (1991), Brouwer e Kleinknecht (1997) que afirmam que MPEs possuem atividades inovativas, muitas vezes não formalizadas, o que tende a diminuir a sua medida de inovação.

A análise das demais empresas, está representada na Figura 2.

Figura 2 - Octógono da Inovação Agrupamento 2

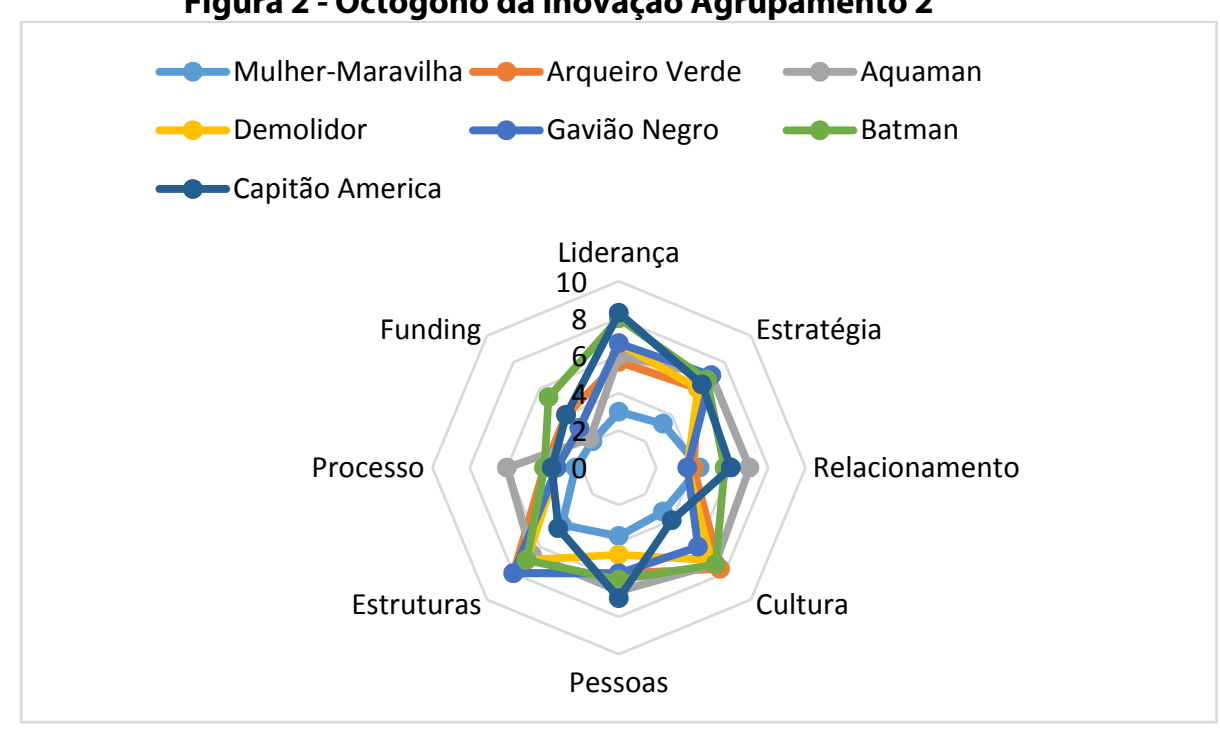

Fonte: Elaborado pelos autores (2016).

Os dados destas foram agrupados por formarem gráficos menos arredondados, identificando carências em dimensões do octógono, a saber: funding, processo, estruturas, pessoas, cultura, relacionamento e estratégia. A empresa Aquaman conta com 25 colaboradores e faturamento entre R\$ 500.001 e R\$ 1.200.000, as demais têm entre 1 e 10 colaboradores e faturamento de até $\mathrm{R} \$ \mathbf{2 4 0 . 0 0 0}$ mil. Três destas estão em estágio de disseminação, duas como parcerias, uma como estruturação e uma com inovação como estratégia. É possível verificar que a empresa MulherMaravilha apresenta dificuldades em todas as dimensões.

A observação dos estágios de inovação indicam que empresas com maior número de colaboradores e faturamento apresentam-se em estágios de inovação mais elevados, corroborando com o estudo PINTEC (2011) que indica um maior nível de inovação em empresas maiores, no entanto, empresas com números menores de 
colaboradores e faturamento são classificadas nos mesmos estágios de inovação das maiores concordando com Kleinknecht, (1987), Kleinknecht e Reijenen (1991), Brouwer e Kleinknecht (1997). Quanto ao estagiamento, identificase a posição da empresa perante à gestão da inovação e o estágio seguinte a ser focado para alcançar o nível de empresa inovadora. No entanto, é preciso levar em conta quais dimensões da inovação devem ser melhoradas para atingir o resultado esperado, é importante então analisar cada dimensão da inovação, proposta por Scherer e Carlomagno (2009), que deram origem aos octógonos da inovação das empresas participantes.

A primeira dimensão a ser discutida é a inovação como processo, em termos acadêmicos e empresariais, pois o entendimento de que é preciso inovar não é suficiente, é preciso compreender a inovação como processo a ser gerenciado. Se observa que todas as empresas encontram dificuldades na gestão de forma que haja inovação. Para Tidd, Bessant e Pavitt (2009) a gestão da inovação é compreendida como planejamento, organização e coordenação de fatores primordiais para desenvolvimento e inovação. Assim, a inovação deve ser tratada como processo que deve envolver diferentes níveis hierárquicos e de conhecimento, vista a necessidade de proposição, acompanhamento e validação de ideias e projetos, assim, o processo de inovação perpassa a organização como um todo e deve estar alicerçada em diferentes dimensões organizacionais (SCHENATTO, 2012; TIDD; BESSANT; PAVITT, 2005, 2009; MEI, 2011; CNI, 2013; NAGANO; PAVANELLI; STEFANOVITZ, 2014; OSENIEKS; BABAUSKA, 2014). O processo inovativo deve ser observado como parte formativa da economia e a discussão do seu financiamento não pode ser abstraída do sistema financeiro e do Estado, pois sistema financeiro e inovação não podem ser dissociados.

A dimensão com destaque como fraqueza nos resultados se relaciona com a forma de investimento em inovação que, por ser diferenciada, tem duas modalidades: o funding e o finance. Durante a fase inicial de um projeto de inovação, o nível de incerteza é alto e o investimento é, por vezes, realizado com fontes internas de recursos. No entanto, para empresas de pequeno porte, onde se enquadram todas as empresas desse estudo, é fundamental que exista crédito oferecido por fontes públicas com custo acessível. Além disso, a inovação vem dos processos da organização que estão diretamente ligados à estrutura organizacional de forma a buscar competitividade, evolução e sustentabilidade.

Com relação a dimensão estrutura, que foi identificada como fraqueza em algumas empresas desse estudo, aponta-se que para que a plena inovação aconteça há necessidade de mudança na estrutura organizacional (TERRA, 2006), de forma a torná-la uma organização mais permeável, porém o que se observa nessas empresas, que nasceram a partir de uma demanda, como característica geral, é a rigidez organizacional que acaba por dificultar mudanças e a inovação (BURN; STALKER, 1961 apud BARBIERI, 2003). Cabe ainda aos colaboradores destas empresas o papel principal no desenvolvimento da inovação, visto que a melhor estrutura não é suficiente se as pessoas não estiverem preparadas e estimuladas a inovar.

A dimensão pessoas, também exige fortalecimento em algumas empresas, sendo que uma vantagem competitiva é baseada no capital humano, sendo este difícil de ser imitado ou copiado, algo individual e coletivo que leva tempo para ser construído (TERRA, 2001). Nas empresas de TI, é necessário articular o capital humano de forma a auxiliar a criação de soluções inovadoras, e esta importância consiste da utilização e combinação de variadas formas de conhecimento, desenvolvendo competências e capacidade inovadora, resultando em novos produtos, processos, sistemas e liderança de mercado, sendo importante o desenvolvimento de uma cultura inovadora onde o gestor reconheça o grupo que faz inovação, pois este necessita de inspiração, proteção, nutrição e provimento de autonomia e recursos (TERRA, 2001).

As empresas carecem de rede de relacionamento, sendo as capacidades inovativas de pequenas empresas alavancadas por contatos locais com negócios, agências de fomento e instituições provedoras de recursos. A dificuldade é estabelecer uma comunidade de empresas que competem na mesma atividade ou base de cliente e, ao mesmo tempo, compartilhem interesses. O fato das empresas participantes desse estudo integrarem o núcleo empresarial, apesar de algumas apresentarem fraquezas na dimensão de relacionamento, contribui para melhores níveis de inovação indo de encontro com os estudos de Balestrin (2005), Verschoore Filho e Balestrin (2006), Steglich (2007) e reafirmando Scherer e Carlomagno (2009, p. 30) que indicam que "a velocidade ou a aceleração com que os conhecimentos novos ultrapassam os anteriores impossibilita que uma empresa sozinha consiga acompanhar a evolução. Os desafios são vencidos através de alianças e parcerias".

Outra dimensão da inovação importante para a organização é a cultura. Uma organização que propicia inovação tem fluidez, ideias que percorrem todos seus setores independentemente do nível hierárquico, além disso, são vistas como conectadas, tem base forte em redes, equipes multifuncionais e camaradagem (JONASH; SOMMERLATT, 1999).

A cultura organizacional é constituída por elementos que tem estreita conexão com seus membros e seus comportamentos, e é a alta gestão que pode interferir nesses artefatos, modificando estruturas e pessoas, 
favorecendo mudanças nas relações entre pessoas e seus comportamentos, criando um ambiente que estimule a inovação. A cultura organizacional influencia diretamente a inovação, uma empresa com canais de comunicação abertos, trabalho em equipe e redes de relacionamento apresenta maior facilidade em estabelecer inovação (SCHERER; CARLOMAGNO, 2009). Então, quando falamos em uma cultura organizacional voltada à inovação é primordial o comprometimento dos líderes.

Com relação a dimensão liderança, a habilidade com gestão de pessoas é diferencial competitivo no âmbito organizacional, havendo hoje maior preocupação com motivação e rotatividade dos colaboradores, estando a motivação ligada diretamente à criatividade e produtividade, gerando por consequência o lucro.

Por fim, a dimensão estratégia, cujo fortalecimento implica na condução de um processo contínuo de decisões que resultam ações e direcionam a organização. Para que a inovação ocorra e seja eficiente nos resultados são necessários o alinhamento da estratégia de negócios e a estratégia de inovação. Estabelecer um direcionamento é eficaz na captação de oportunidades de inovação, pois empresas mais inovadoras adotam diferentes temas a fim de guiar as ações de colaboradores e parceiros, o que forma uma infraestrutura básica e provê um caminho inicial para inovação (SCHERER; CARLOMAGNO, 2009).

Observa-se então, que apesar de cada empresa apresentar certas dimensões como mais fracas que outras, devido à complexidade da inovação, ações de fortalecimento são importantes na busca por um estágio maior de inovação.

\section{CONCLUSÃO}

A partir dos dados coletados quanto ao estágio da inovação, propiciado pelo instrumento proposto por Carvalho, Reis e Cavalcante (2011), e pelo diagnóstico Octógono da Inovação de Scherer e Carlomagno (2009), é evidente que duas das empresas com maior número de colaboradores e maior faturamento apresentam estágios da inovação maiores, indo de encontro com seus octógonos da inovação, indicando constância de suas dimensões, havendo melhorias a ser implementadas de forma a alcançar estágios maiores de inovação .

As demais empresas, em referência ao estágio da inovação oscilam entre Estruturação e Disseminação. A análise dos dados da empresa Aquaman, destaca fraqueza no financiamento da inovação ou funding, pode ter como solução a busca de investimento privado ou público. O quadro da empresa Ciborgue, indica que, com poucas melhorias e estratégias de fortalecimento, apresenta potencial de obtenção de estágios maiores de inovação. Já a empresa Mulher-Maravilha, apresenta fraquezas em todas as dimensões no octógono da inovação. Todas as dimensões da inovação são complementares e de grande relevância no processo inovativo, assim, é importante o planejamento de ações de fortalecimento de forma abrangente e proativa, pensando no futuro da organização, em suas metas e da inovação como um ciclo ininterrupto.

Observa-se como limitação desse estudo, por ser multicasos, sua abrangência, orienta-se então a aplicação de uma metodologia de avaliação da inovação específica para o setor de Tl de forma a fazer inferências ao setor como um todo.

Se ressalta a necessidade, em trabalhos futuros, da verificação e proposição de melhorias para as dimensões defasadas, assim como, metas e ações para aumento do desempenho dos constructos e dimensões analisadas. Também, para tornar o processo análise mais dinâmico, há oportunidade de construção de software que ofereça facilidade ao gestor ou aos pesquisadores na aplicação de métodos, na mensuração dos resultados e no direcionamento das ações.

Desta forma, destaca-se a importância deste tipo de diagnóstico para a continuidade e fomento da inovação, melhorias no desempenho e competitividade das empresas do setor de tecnologia da informação. A importância da aplicação de um diagnóstico da inovação é clara, principalmente em um setor da economia em franco crescimento com necessidade de ser competitivo e inovador em um mercado cada vez mais agressivo.

\section{REFERÊNCIAS}

BABBIE, E R. Métodos de pesquisas de survey. Belo Horizonte: Editora da UFMG, 1999.

BALESTRIN, A. A dinâmica da complementariedade de conhecimentos no contexto das redes

interorganizacionais. 214f. Tese (Programa de Pós-Graduação em Administração) - Universidade Federal do Rio Grande do Sul, Porto Alegre, 2005.

BARBIERI, J. C. (Org.). Organizações inovadoras: estudos e casos brasileiros. Rio de Janeiro: FGV, 2003 
BARBIERI, J. C.; ÁLVARES, A. C. Inovações nas Organizações Empresariais. In: BARBIERI, J.C. (Org.). Organizações Inovadoras: estudos e casos brasileiros. Rio de Janeiro: Editora FGV, 2004. p. 41-64.

BACHMANN, D. L.; DESTEFANI, J. H. Proposal for Assessment of Innovation Degree at Small Companies. SEMINÁRIO NACIONAL DE PARQUES TECNOLÓGICOS E INCUBADORAS DE EMPRESAS, 18., Aracaju. Anais... Aracaju: ANPROTEC, 2008.

BNDES. Porte da Empresa. 2011 Disponível em: <http://www.abic.com.br/publique/media/portedeempresa.pdf> Acesso em: 13 jan. 2016.

BRESCHI, S.; MALERBA, F. Sectoral Innovation Systems: technological regimes, schumpeterian dynamics, and spacial boundaries. In: EDQUIST, C. (Ed.). Systems of innovation: technologies, institutions, and organizations. London: Pinter, 1997. p. 130-156.

BROUWER E.; KLEINKNECHT A. Measuring the unmeasurable: a country's non-R\&D expenditure on product and service innovation. Research Policy, v. 25, p. 1235-1242, 1997.

CARON, A. Inovação tecnológica em pequenas e médias empresas. Revista FAE BUSINESS, n. 8, p. 25-28, 2004.

CARVALHO, H. G.; REIS, D. R.; CAVALCANTE, M. B. Gestão da Inovação. Curitiba: Aymará, 2011

CONFEDERAÇÃO NACIONAL DA INDÚSTRIA (CNI). Mapa estratégico da indústria 2013-2022. Brasília: CNI, 2013.

CHESBROUGH, H.W. The era of open innovation. MIT Sloan Management Review, v. 44, n. 3, p. 33-41, 2003.

CHESBROUGH, H.W. Open business models: how to thrive in the new innovation landscape. Boston: Harvard Business School Press, 2006.

DUARTE, C. H. C.; BRANCO, C. E. Impactos econômicos e sociais da política setorial brasileira para tecnologias da informação. Revista do BNDES, Rio de Janeiro, v. 8, n. 15, p. 125-146, 2001

DUARTE, V. C.; FERRAZ FILHO, G. O programa SOFTEX e a construção da indústria de software nacional. Revista Brasileira de Comércio Exterior, Rio de Janeiro, v. 13, n. 56, p. 23-30, 1998.

FRANK, A. G. et al. The effect of innovation activities on innovation outputs in the Brazilian industry: Marketorientation vs. technology-acquisition strategies. Research Policy, v. 45, n. 3, p. 577-592, 2016.

GRILICHES, Z. Patent Statistics an Economic Indicator: Survey part I. NBER Working Paper Series, Working Paper no. 3301, Cambridge: National Bureau of Economic Research, 1990.

GRIZENDI, E. Manual de inovação para empresas brasileiras de TIC: orientações gerais sobre inovação para empresas do setor de tecnologia da informação e comunicação. Rio de Janeiro: Publit, 2012.

INSTITUTO NACIONAL DE EMPREENDEDORISMO E INOVAÇÃO (INEI). Avaliação do Grau de Inovação

Organizacional - QDI. Disponível em: <http://www.inei.org.br/produtos-eservicos/qdi>. Acesso em: 27 jan. 2016.

JONASH, R. S.; SOMMERLATTE, T. O Valor da Inovação: (the innovation Premium) Como as Empresas Mais Avançadas Atingem Alto desempenho e Lucratividade. Rio de Janeiro: Campus, 2001.

KLEINKNECHT, A. Measuring R\&D in small firms: How much we are missing? Journal of Industrial Economics, v. 36, p. 253-256, 1987.

KLEINKNECHT, A.; REIJNEN, J. O. N. More evidence on the undercounting of small firm R\&D. Research Policy, v. 20, p. 579-587, 1991.

KNOX, S. The boardroom agenda: Developing the innovative organization. Corporate Governance, Bradford, UK, v. 2, n. 1, p. 27-36, 2002.

MACKINNON, L. The 7 Dimensions of Innovation . Disponível em: <http://www.think-differently.org/2007/05/7dimensions-of-innovation.html>. Acesso em: 27 jan. 2016. 
MALERBA, F.; ORSENIGO, L. Technological regimes and firm behaviour. Industrial and corporate change. Oxford University Press, v. 2, n. 1, p. 45-71, 1993.

MARQUES, C. A. N.; SUZUKI, J. A.; FARIA, A. F. Modelo de estudo de viabilidade aplicado a empresas de software. In: ENCONTRO NACIONAL DE ENGENHARIA DE PRODUÇÃO, 30., São Carlos, 2010. Anais... São Paulo: ENANPAD, 2010. p. 12-15.

MOBILIZAÇÃO EMPRESARIAL PELA INOVAÇÃO (MEI). Estratégia e objetivos. 2011. Disponível em: $<$ http://inova.sistemafindes.org.br/Arquivos/Downloads/18/MEl\%20- \%20Estrategias\%20e\%20Objetivos.pdf>. Acesso em: 16 jan. 2016.

MILESI, D.; PETELSKI, N.; VERRE, V. Innovation and appropriation mechanisms: Evidence from Argentine microdata. Technovation, v. 33, n. 2, p. 78-87, 2013.

MIOZZO, M. et al. Innovation collaboration and appropriability by knowledge-intensive business services firms. Research Policy, v. 45, n. 7, p. 1337-1351, 2016.

MIOZZO, M; SOETE, L. Internationalization of Services: A Technological Perspective. Technological Forecasting and Social Change, n. 67, p. 159-185, 2001.

MYTELKA, L. A role for innovation networking in the other two-thirds. Futures, v. 85, n. 1, p. 694-712, 1993.

NAGANO, M. S.; STEFANOVITZ, J. P.; VICK, T. E. Innovation management processes, their internal organizational elements and contextual factors: an investigation in Brazil. Journal of Engineering and Technology Management, v. 33, p. 63-92, 2014.

OCDE. Manual de Oslo - Diretrizes para coleta e interpretação de dados sobre inovação. Brasília: FINEP, 2006.

OSENIEKS, J.; BABAUSKA, S. The relevance of innovation management as prerequisite for durable existence of small and medium enterprises. Procedia - Social and Behavioral Sciences, v. 110, p. 82-92, 2014.

PINTEC. Banco de dados da Pesquisa Industrial de Inovação Tecnológica. 2011. Disponível em: <http://www.pintec.ibge.gov.br/index.php?option=com_content\&view category\&layout=blog\&id=15\&ltemid=19> Acesso em: 27 jan. 2016.

SAWHNEY, M. et al. The 12 Different Ways for Companies to Innovate. MIT Sloan Management Review, p. 75-81, 2006.

SHAPIRO, S. Innovation: A Blueprint for Surviving and Thriving in an Age of Change. USA: McGraw-Hill Companies, 2001.

SCHENATTO, F. J. A. Estratégia tecnológica para arranjos Produtivos locais: uma metodologia baseada na elaboração de estudos prospectivos. 2012. Dissertação (Mestrado em Engenharia de Produção) - Universidade Federal de Santa Catarina, Florianópolis, 2012.

SCHERER, F. O.; CARLOMAGNO, M. S. Gestão da Inovação na Prática: como aplicar conceitos e ferramentas para alavancar a inovação. São Paulo: Atlas, 2009.

SCHUMPETER, J. A. The theory of Economic Developement. Cambridge: Harvard University Press, 1934.

SILVA, C. G.; MELO, L. C. P. Ciência, tecnologia e inovação: desafios para a sociedade brasileira - livro verde. Brasília: Ministério da Ciência e Tecnologia, 2001.

SOUZA BERMEJO, P. H. et al. Conceptualizing organizational innovation: The case of the Brazilian software industry. Information \& Management, v. 53, n. 4, p. 493-503, jun. 2015.

STEFANOVITZ, J. P.; NAGANO, M. S Gestão da inovação: análise e síntese dos conceitos. Produto \& Produção, v. 15, n. 2, p. 11-23, 2014.

STEGLICH, L.R. O desenvolvimento de produtos em uma rede de pequenas e médias empresas: motivações e fatores críticos. 2007, 96f. Dissertação (Programa de Pós-Graduação em Administração) - Universidade Federal do Rio Grande do Sul, Porto Alegre, 2007. 
SUH, D.; HWANG, J. An analysis of the effect of software intellectual property rights on the performance of software firms in South Korea. Technovation, v. 30, n. 5, p. 376-385, 2010.

TERRA, B. Em Tempos de Rede: A Gestão do Conhecimento para o Desenvolvimento de Regiões. Rio de Janeiro: Interciência, 2006.

TERRA, J. C. C. Gestão do Conhecimento: o grande desafio empresarial. São Paulo: Negócio Editora, 2001.

TIDD, J.; BESSANT, J.; PAVITT, K. Gestão da Inovação. 3. ed. Porto Alegre: Bookman, 2009.

TIDD, J.; BESSANT, J.; PAVITT, K. Managing innovation, integration technological, market and organizational change. West Sussex: John Wiley \& Sons, 2005.

VAN DE VEN, A. H. et al. The Innovation Journey. New York: Oxford University Press, 1999.

VAONA, A.; PIANTA, M. Firm Size and Innovation in European Manufacturing. Small Business Economics, v. 30, p. 283-299, 2008.

VEE, Annette. Carving up the commons: How software patents are impacting our digital composition environments. Computers and Composition, v. 27, n. 3, p. 179-192, 2010.

VERGARA, S. C. Projetos e relatórios de pesquisa em administração. São Paulo: Atlas, 2000.

VERSCHOORE FILHO, J.; BALESTRIN, A. Competitive factors of cooperation networks: a quantitative study of a southern brazilian case. EUROPEAN GROUP FOR ORGANIZATION STUDIES COLLOQUIUM, 22., 2006.

Proceedings....Bergen: [s.n.], 2006.

XAVIER SOBRINHO, G. G. de F. Perfil e perspectivas do trabalho no complexo das tecnologias de informação: a produção de softwares no Rio Grande do Sul. Ensaios FEE, Porto Alegre, v. 21, n. 2, p. 77-109, 2000.

\section{ANEXO A - DIAGNÓSTICO OCTÓGONO DA INOVAÇÃO}

Pontue cada afirmativa com notas entre 1 (menor nota) e 9 (maior nota).

\begin{tabular}{|c|c|c|}
\hline \multicolumn{3}{|c|}{ Diagnóstico octógono da inovação } \\
\hline No & Afirmativas & Aval. \\
\hline 1 & Nossas iniciativas de inovação estão logicamente alinhadas com a estratégia da empresa. & \\
\hline 2 & $\begin{array}{l}\text { Iniciativas altamente inovadoras são tratadas fora da estrutura organizacional principal, em } \\
\text { projetos específicos. }\end{array}$ & \\
\hline 3 & Disponibilizamos tempo, benefícios e incentivos (financeiros e não financeiros) para a inovação. & \\
\hline 4 & Todos dentro da empresa compreendem a necessidade de inovação. & \\
\hline 5 & $\begin{array}{l}\text { Nossa estrutura organizacional é flexível, com poucos níveis hierárquicos e muita } \\
\text { descentralização nas decisões. }\end{array}$ & \\
\hline 6 & Apresentamos foco claro no crescimento por meio da inovação. & \\
\hline 7 & Permitimos que todos corram riscos e cometam erros na busca por novas soluções. & \\
\hline 8 & Iniciativas e atividades de inovação seguem uma coordenação definida. & \\
\hline 9 & As lideranças da empresa têm clareza sobre o conceito e a importância da inovação. & \\
\hline 10 & Temos um orçamento específico para os projetos de inovação. & \\
\hline 11 & Utilizamos ferramentas de gestão de projetos para conduzir as iniciativas de inovação. & \\
\hline 12 & $\begin{array}{l}\text { Novas ideias são avaliadas conforme medidas de desempenho que levam em consideração } \\
\text { riscos e incertezas. }\end{array}$ & \\
\hline 13 & $\begin{array}{l}\text { O desempenho das lideranças é avaliado com métricas específicas relacionadas a sua atuação } \\
\text { no processo de Gestão da Inovação. }\end{array}$ & \\
\hline 14 & $\begin{array}{l}\text { Conhecimentos e ferramentas necessárias para inovar são do conhecimento de todos dentro da } \\
\text { organização. }\end{array}$ & \\
\hline
\end{tabular}




\begin{tabular}{|c|c|}
\hline 15 & $\begin{array}{l}\text { Utilizamos nossas redes de clientes, fornecedores e até concorrentes para gerar e refinar novas } \\
\text { ideias. }\end{array}$ \\
\hline 16 & As lideranças dedicam tempo e atenção ao acompanhamento dos projetos de inovação. \\
\hline 17 & Temos temas, objetivos e metas definidos para inovação. \\
\hline 18 & Avaliamos sistematicamente os resultados das iniciativas de inovação. \\
\hline 19 & $\begin{array}{l}\text { Conhecemos profundamente as necessidades reveladas e não reveladas de nossos clientes e } \\
\text { não clientes. }\end{array}$ \\
\hline 20 & Dispomos de um processo estruturado para geração e seleção das melhores ideias. \\
\hline 21 & $\begin{array}{l}\text { Temos um processo sistemático para acompanhamento de novas tendências de mercado e } \\
\text { tecnologias. }\end{array}$ \\
\hline 22 & Utilizamos mecanismos de comunicação para fomentar a inovação na empresa. \\
\hline 23 & Nossa equipe apresenta alta diversidade de conhecimentos, valores, carreira e interesses. \\
\hline 24 & $\begin{array}{l}\text { Buscamos recursos financeiros para inovação em diferentes fontes (capital de risco, órgãos de } \\
\text { governo, parceiros etc.) }\end{array}$ \\
\hline
\end{tabular}

Fonte: Scherer e Carlomagno (2009).

\section{ANEXO B - FORMULÁRIOS DE AVALIAÇÃO DO ESTÁGIO DE INOVAÇÃO}

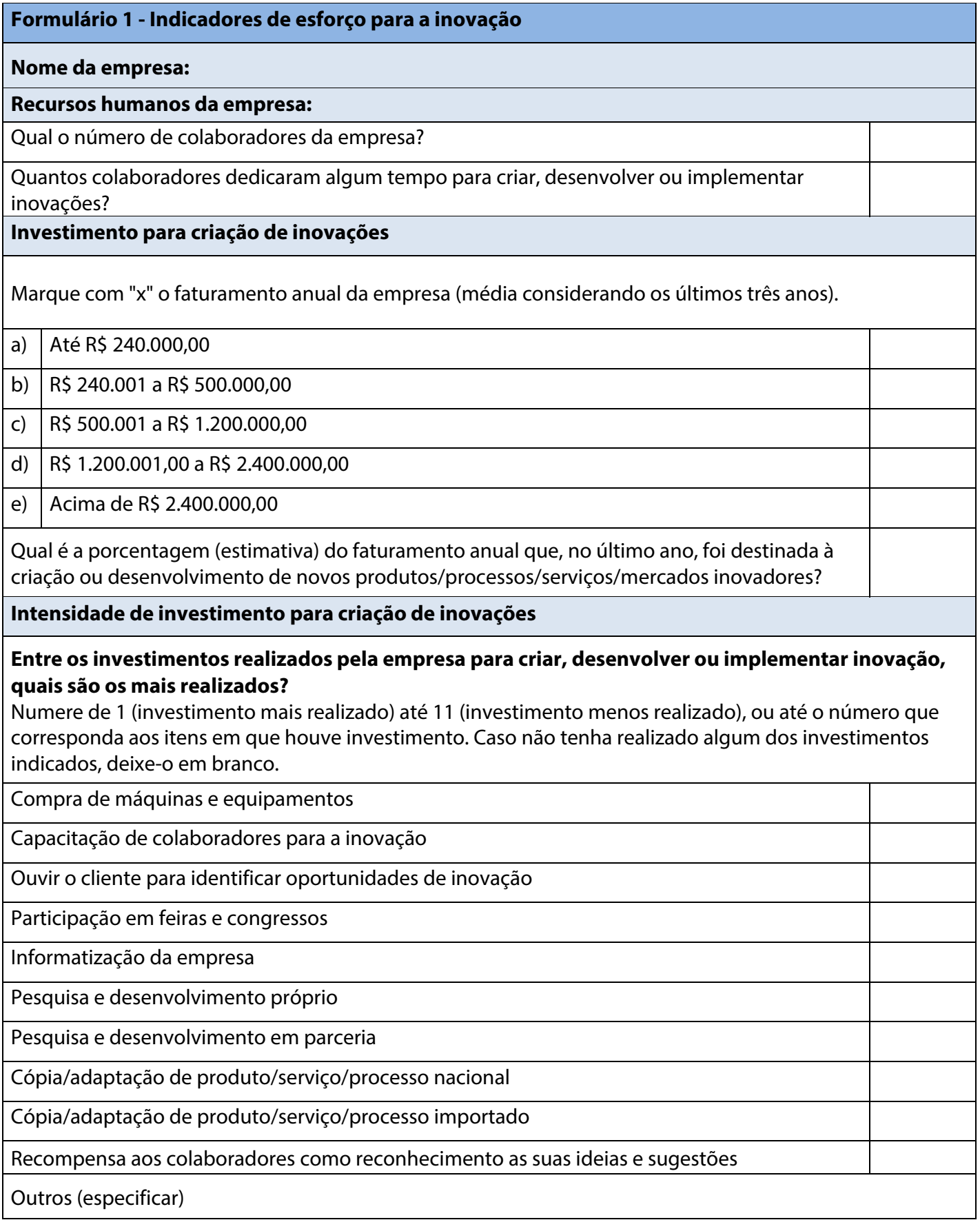




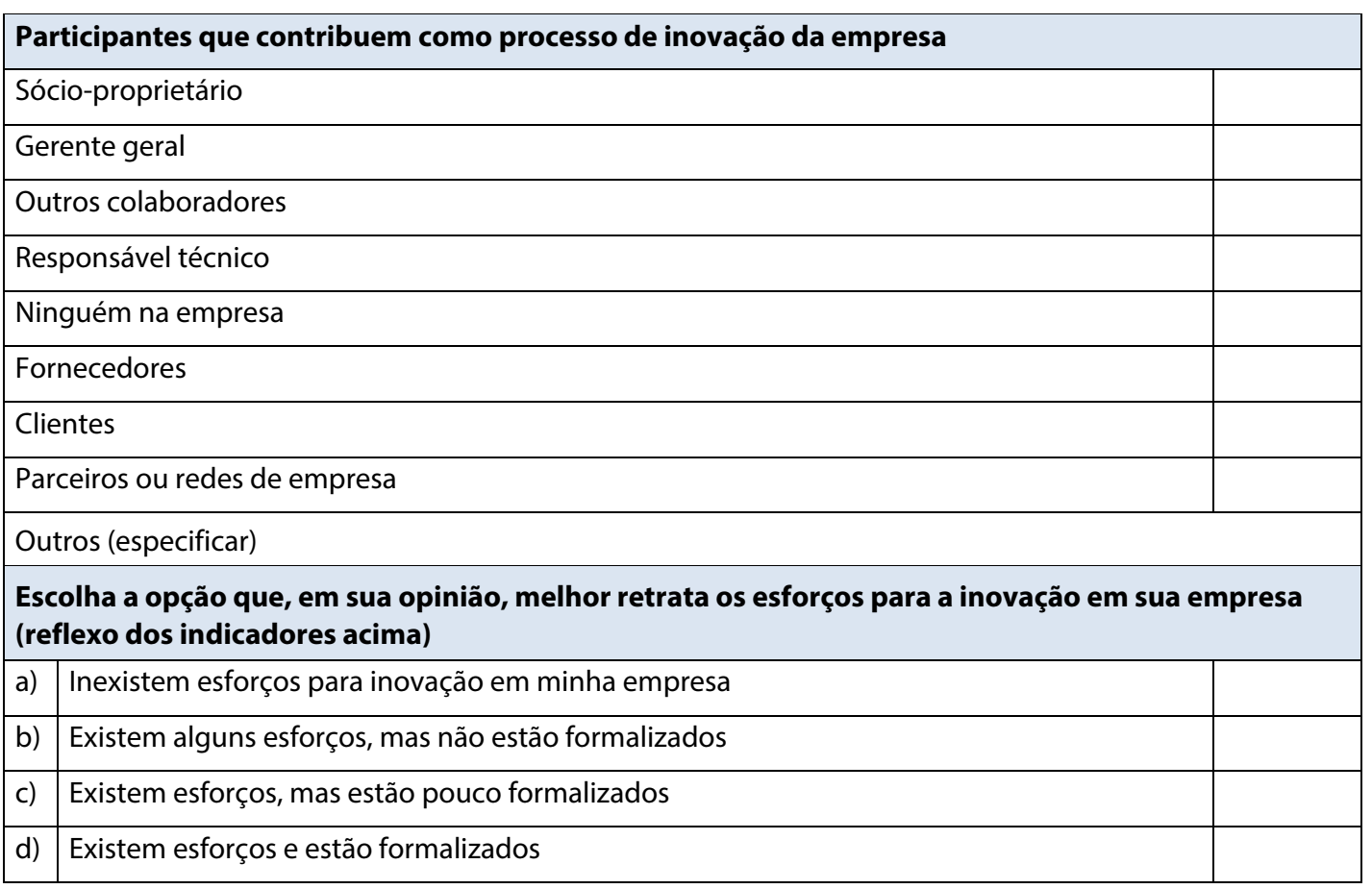

Fonte: Carvalho, Reis e Cavalcante (2011)

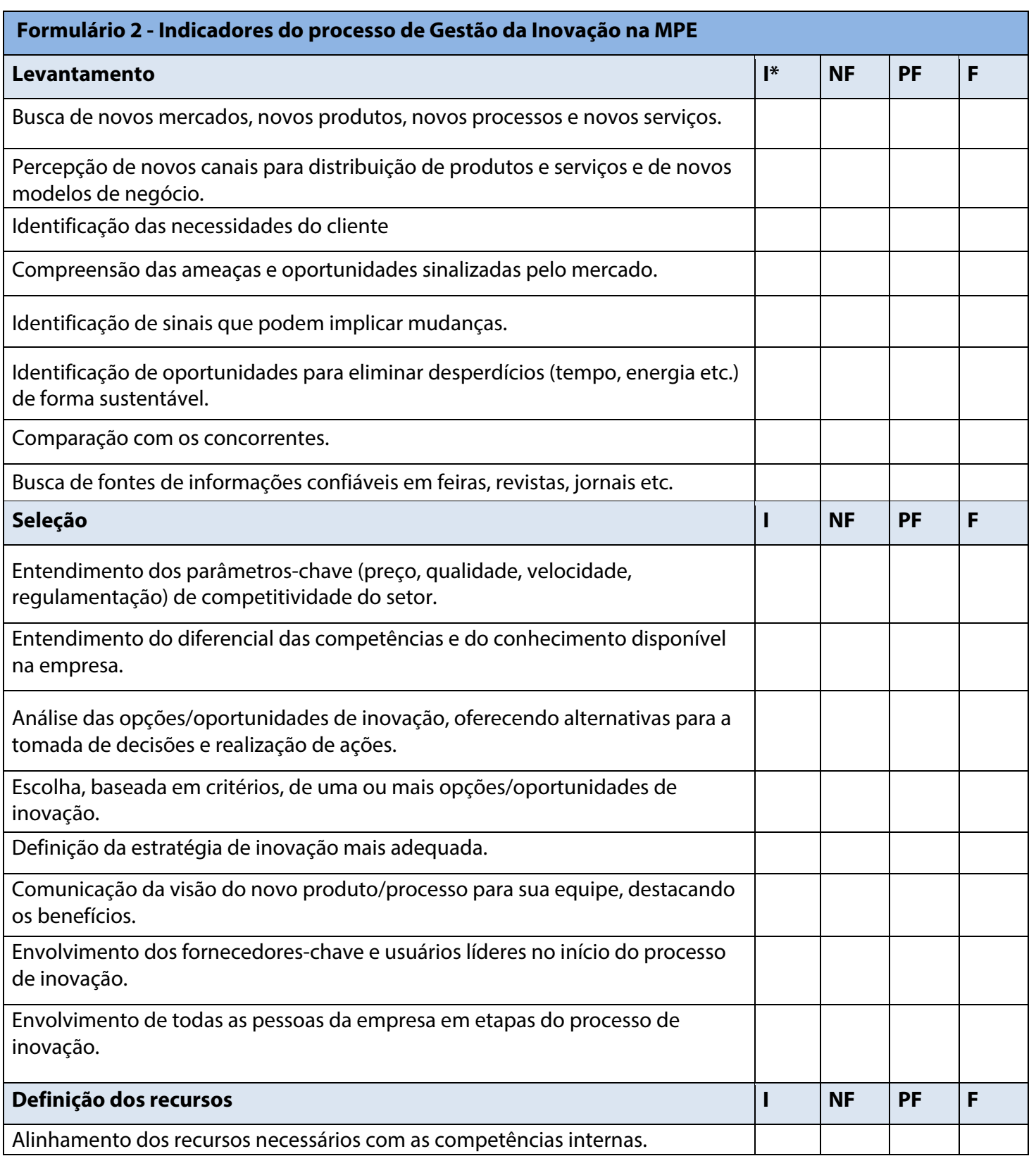




\begin{tabular}{|c|c|c|c|c|}
\hline Realização da compra, licenciamento, contratação e novidades fora da empresa. & & & & \\
\hline $\begin{array}{l}\text { Identificação das formas de acesso aos recursos, por exemplo, compra de } \\
\text { insumos, tecnologias e serviços; parcerias com institutos, universidades ou } \\
\text { centros de excelência; cópia, contratação de especialistas; associações e alianças } \\
\text { estratégicas etc. }\end{array}$ & & & & \\
\hline Implementação & I & NF & PF & $\mathbf{F}$ \\
\hline $\begin{array}{l}\text { Definição do escopo do projeto da inovação a ser introduzida e/ou } \\
\text { implementada. }\end{array}$ & & & & \\
\hline $\begin{array}{l}\text { Estabelecimento de datas e formas de acompanhamento (prazo, custo e } \\
\text { qualidade). }\end{array}$ & & & & \\
\hline Aquisição de recursos previstos. & & & & \\
\hline Preparação do lançamento da inovação. & & & & \\
\hline Compartilhamento da visão com os demais funcionários. & & & & \\
\hline $\begin{array}{l}\text { Alinhamento das atividades de introdução da inovação com todos os } \\
\text { funcionários. }\end{array}$ & & & & \\
\hline Aprendizagem & I & NF & PF & $\mathbf{F}$ \\
\hline Reflexão sobre o processo de inovação como um todo. & & & & \\
\hline Registro das lições aprendidas, evitando "reinventar a roda". & & & & \\
\hline Utilização de aprendizado já obtido no lançamento de produtos/serviços. & & & & \\
\hline Compartilhamento da experiência adquirida com os colaboradores & & & & \\
\hline $\begin{array}{l}\text { Utilização de novas formas de disseminação de informação e conhecimento na } \\
\text { empresa. }\end{array}$ & & & & \\
\hline SOMATÓRIO & & & & \\
\hline
\end{tabular}

Escolha a opção que, em sua opinião, melhor retrata o processo de inovação como um todo em sua empresa, observando as respostas com maior incidência.

\begin{tabular}{|l|l|l|}
\hline a) & Inexiste um processo de inovação em minha empresa. & \\
\hline b) & Existem algumas atividades das etapas, mas não estão formalizadas. & \\
\hline c) & Existem diversas atividades das etapas, mas estão pouco formalizadas. & \\
\hline d) & Existe um processo de inovação e está formalizado. & \\
\hline
\end{tabular}

*I: inexiste / F: Não formalizado / PF: Pouco formalizado / F: Formalizado Fonte: Carvalho, Reis e Cavalcante (2011)

\begin{tabular}{|c|c|c|c|c|}
\hline \multicolumn{5}{|l|}{ Formulário 3 - Indicadores de práticas de estímulo à inovação } \\
\hline \multicolumn{5}{|c|}{$\begin{array}{l}\text { Assinale com "x" a alternativa que melhor representa a realidade de sua empresa e some o número de vezes } \\
\text { em que cada item é assinalado. }\end{array}$} \\
\hline Criatividade & *I & NF & PF & $\mathbf{F}$ \\
\hline \multicolumn{5}{|l|}{$\begin{array}{l}\text { Apoia a transformação de ideias em prática, por meio da resolução criativa de } \\
\text { problemas, favorecendo a busca de novas perspectivas. }\end{array}$} \\
\hline Processos de comunicação & I & NF & PF & $\mathbf{F}$ \\
\hline \multicolumn{5}{|l|}{$\begin{array}{l}\text { Possibilita captar informações e levá-las para dentro da empresa, ou da empresa } \\
\text { para seus clientes e fornecedores, sob uma perspectiva compartilhada e } \\
\text { transparente. }\end{array}$} \\
\hline Reconhecimento e recompensa & I & NF & PF & $\mathbf{F}$ \\
\hline \multicolumn{5}{|l|}{$\begin{array}{l}\text { Possibilita valorizar os colaboradores que equalizam seus interesses profissionais } \\
\text { com os interesses estratégicos da empresa, de forma objetiva e alinhada com a } \\
\text { personalidade e cultura da organização. }\end{array}$} \\
\hline Capacitação de recursos humanos & I & NF & PF & $\mathbf{F}$ \\
\hline $\begin{array}{l}\text { Amplia a capacidade dos colaboradores de fazer melhor uso dos recursos da } \\
\text { empresa (infraestrutura, pessoas, sistemas de TI etc.). }\end{array}$ & & & & \\
\hline
\end{tabular}


Evolui a capacidade da empresa em desenvolver/produzir produtos e serviços com inovação em design, qualidade, desempenho etc.

\section{SOMATÓRIO}

Escolha a opção que, em sua opinião, melhor retrata a existência de práticas de estímulo à inovação em sua empresa.

\begin{tabular}{|l|l|l|}
\hline a) & Inexistem práticas de estímulo à inovação em minha empresa. & \\
\hline b) & Existem algumas práticas, mas não estão formalizadas. & \\
\hline c) & Existem diversas práticas, mas pouco formalizadas. & \\
\hline d) & Existem todas as práticas de estímulo à inovação e estão formalizadas. & \\
\hline
\end{tabular}

*I: inexiste / F: Não formalizado / PF: Pouco formalizado / F: Formalizado

Fonte: Carvalho, Reis e Cavalcante (2011)

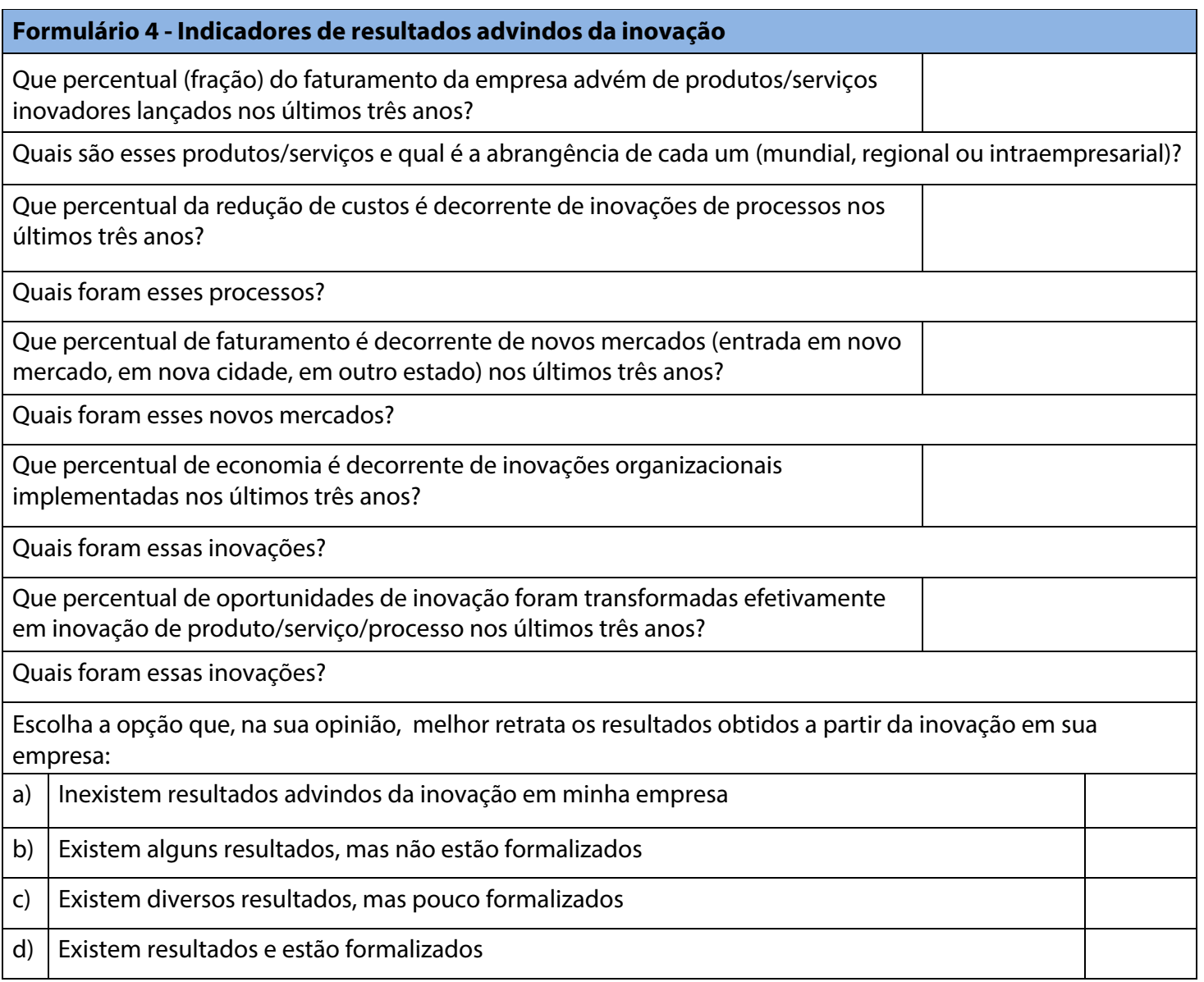

Fonte: Carvalho, Reis e Cavalcante (2011)

\begin{tabular}{|c|c|c|c|c|c|c|c|c|}
\hline \multicolumn{9}{|c|}{ Formulário 5 - Rumo à empresa inovadora } \\
\hline & & \multicolumn{7}{|c|}{ Autoavaliação } \\
\hline \multicolumn{2}{|c|}{ Escala } & Esforços & Processos & Estímulos & Resultados & $S$ & $P$ & $\mathrm{R}$ \\
\hline a) & Inexistente (s) & & & & & & 0 & \\
\hline b) & Não formalizado (s) & & & & & & 1 & \\
\hline c) & Pouco formalizado (s) & & & & & & 3 & \\
\hline d) & Formalizado (s) & & & & & & 5 & \\
\hline \multicolumn{6}{|c|}{ SOMATÓRIO } & & & \\
\hline
\end{tabular}

Fonte: Carvalho, Reis e Cavalcante (2011) 